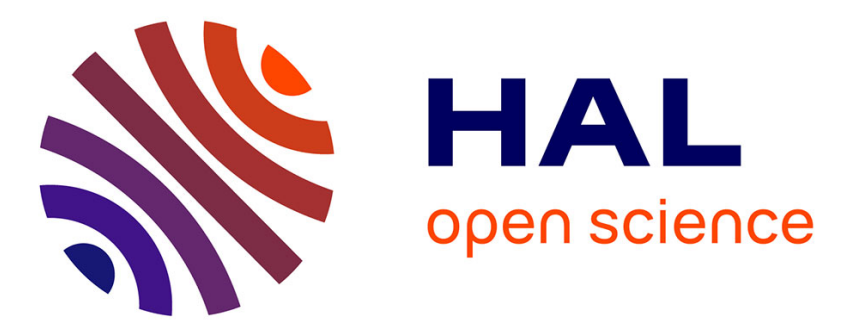

\title{
Cooperative localization of vehicles sharing GNSS pseudoranges corrections with no base station using set inversion
}

Khaoula Lassoued, Philippe Bonnifait, Isabelle Fantoni

\section{To cite this version:}

Khaoula Lassoued, Philippe Bonnifait, Isabelle Fantoni. Cooperative localization of vehicles sharing GNSS pseudoranges corrections with no base station using set inversion. IEEE Intelligent Vehicle Symposium (IV 2016), Jun 2016, Göteborg, Sweden. pp.496-501, 10.1109/IVS.2016.7535432 • hal01369824

\section{HAL Id: hal-01369824 \\ https://hal.science/hal-01369824}

Submitted on 26 Sep 2016

HAL is a multi-disciplinary open access archive for the deposit and dissemination of scientific research documents, whether they are published or not. The documents may come from teaching and research institutions in France or abroad, or from public or private research centers.
L'archive ouverte pluridisciplinaire $\mathbf{H A L}$, est destinée au dépôt et à la diffusion de documents scientifiques de niveau recherche, publiés ou non, émanant des établissements d'enseignement et de recherche français ou étrangers, des laboratoires publics ou privés. 


\title{
Cooperative Localization of Vehicles sharing GNSS Pseudoranges Corrections with no Base Station using Set Inversion
}

\author{
Khaoula Lassoued, Philippe Bonnifait and Isabelle Fantoni
}

\begin{abstract}
Fully distributed localization methods with no central server are relevant for autonomous vehicles that need real-time cooperation. In this paper, mobile vehicles share estimates of GNSS pseudoranges common errors also known as biases. The biases that affect the pseudoranges are mainly due to signal propagation and inaccurate ephemeris data. By describing the measurements models as geometric constraints on intervals, cooperative localization turns into distributed set inversion problem. The solution of this problem is guaranteed to contain the true vehicles positions. We consider vehicles which cooperate and exchange information in order to improve the absolute and relative estimation by fusing pseudoranges corrections shared between them. Results using real measurements are presented to illustrate the performance of the proposed approach in comparison with a standalone method.
\end{abstract}

\section{INTRODUCTION}

In intelligent vehicles systems, reliable position estimates are crucial. Mutual cooperation aims to enhance positioning and reduce uncertainty arising from low cost sensors. For example, when using low cost Global Navigation Satellite Systems (GNSS) receivers in complex environments, atmospheric and ephemeris errors can have a great impact on the estimated position. The effects of these errors can be reduced by considering information exchange. Our target is to eliminate the effects of the slowly varying pseudoranges errors that bias the estimation and to improve the relative and absolute localization performance.

Common approaches for localization are Bayesian methods relying on Extended Kalman Filtering [1] or Least Square Estimation [2]. These probabilistic methods estimate the position but do not provide reliable confidence domains. Set Inversion Via Interval Analysis (SIVIA) (a particular setmembership or bounded-error estimation method) provides an elegant solution to the data incest problem arising from the repetitive fusion of identical information. The SIVIA method is able to give sets that always contain the true position of the vehicles without any over-convergence. A set-membership approach is based on the hypothesis that model and measurement errors are bounded. It has been successfully applied for model parameters estimation [3] and robots position estimation when reliable confidence domains are required [4]. Meizel et al. [5] developed a set inversion method based on bounded-error observers for single robot localization. However, SIVIA is not suitable to be applied in real time if the number of bisections is high. One solution is to use SIVIA while simultaneously solving a Constraint

The authors are with Sorbonne universités, Université de Technologie de Compiègne, CNRS, UMR 7253 Heudiasyc, 60200 Compiègne, France.

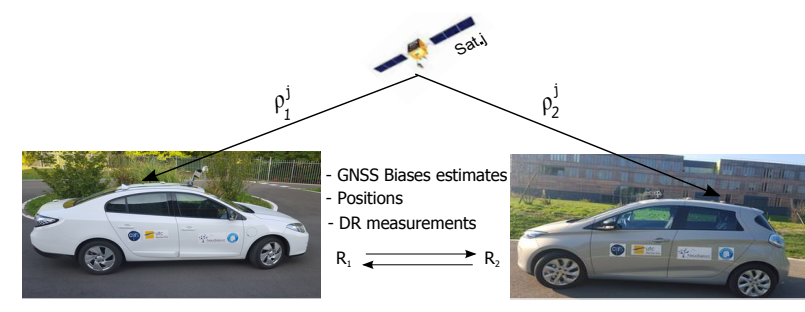

Fig. 1: Experimental vehicles and shared data

Satisfaction Problem (CSP) to limit the computing time of bisections [6]. Concerning multiple robots localization in real time, several recent studies are based on set inversion under CSP. Drevelle et al. [7] exploited a group of Automated Underwater Vehicles (AUVs) to characterize a large explored space for monitoring. In their application, they used range sensors to measure the inter-distance between robots. Moreover, A. Bethencourt [8] used distributed set-membership algorithms in a group of AUVs based on inter-temporal measurements to accomplish a swarm cooperative mission. In this work, the data fusion of Dead Reckoning (DR) and exteroceptive sensors was used but biased measurements were not considered.

In this paper, we propose a new formulation of multi-vehicles cooperation using set inversion with CSP techniques on intervals. We assume that they are able to cooperate and exchange information such that each vehicle can compute the partner position with a reliable domain. The main contribution of this paper is to show the improvement of the egopositioning and of the mutual localization among networked vehicles that share estimated biases (or corrections) of GNSS pseudoranges. The cooperative scenario is illustrated in Fig. 1. The developed method allows the data fusion of estimated biases in a distributed way with no central fusion node and with no base station. Experimental validation with two vehicles is then realized to evaluate the performance in real conditions.

Section II introduces the system modeling and an observability study of the cooperative system. Section III presents the set inversion method using CSP on intervals. The proposed distributed cooperative localization algorithm is described in section IV. Experimental results are presented in section V.

\section{StRuCTURAL PROPERTIES}

The data fusion of GNSS measurements with DR measurements is often adopted to enhance localization accuracy. In this section, the mathematical models of the measurements are described and an observability study of the cooperative system is considered. 


\section{A. Models}

The GNSS raw measurements considered here are $L 1$ pseudoranges. The pseudorange $\rho_{i}^{j}$ [9] is the measure of each vehicle $R_{i}\left(i=\left\{1, \ldots, n_{r}\right\}\right)$ located at coordinates $\boldsymbol{p}_{i}=$ $\left[x_{i}, y_{i}, z_{i}\right]$ to each satellite $j\left(j=1, \ldots, n_{s}\right)$ at position $p^{j}=\left[x^{j}, y^{j}, z^{j}\right]$. We model the common GNSS error $b^{j}$ as an additive error. The model of $\rho_{i}^{j}$ is given by Eq. (1).

$$
\rho_{i}^{j}=\sqrt{\left(x_{i}-x_{j}\right)^{2}+\left(y_{i}-y_{j}\right)^{2}+\left(z_{i}-z_{j}\right)^{2}}+b^{j}+d_{i}+\beta^{j}
$$

where $d_{i}$ represents the receiver clock offset and $\beta^{j}$ the measurement noise. Please refer to [10] for further details.

Let ${ }^{i} \boldsymbol{y} \in \mathbb{R}^{n_{s}}$ be the vector of $n_{s}$ pseudoranges measurements and ${ }^{i} \boldsymbol{x} \in \mathbb{R}^{n}$ be the state vector of vehicle $i$. The observation model at discrete time $k$ is defined as follows:

$$
{ }^{i} \boldsymbol{y}_{k}=\boldsymbol{g}\left({ }^{i} \boldsymbol{x}_{k}\right)
$$

Suppose that each vehicle is equipped with a compass and wheel encoders to measure the yaw angle and the speed. These measurements are seen as an input vector ${ }^{i} \boldsymbol{u}(t)=$ $\left[\begin{array}{cc}v_{i} & \psi_{i}\end{array}\right]^{T}$. A unicycle evolution model for the pose, a linear drift assumption for the clock and an auto-regressive (AR) model for the biases allow expressing the derivative of the state by a non-linear function $f$ in a local East North Up frame:

$$
\boldsymbol{f}\left({ }^{i} \boldsymbol{x},{ }^{i} \boldsymbol{u}\right)=\left\{\begin{array}{l}
\dot{x}_{i}=v_{i} \cos \left(\psi_{i}\right) ; \quad \dot{y}_{i}=v_{i} \sin \left(\psi_{i}\right) ; \quad \dot{z}_{i}=0 \\
\dot{d}_{i}=d r_{i} ; \quad \dot{d r_{i}}=0 ; \quad \dot{b}^{j}=a b^{j}
\end{array}\right.
$$

$\psi_{i}$ is the orientation angle of the vehicle assumed to be measured. $v_{i}$ represents the measured linear velocity of the vehicle. $d_{i}$ and $d r_{i}$ are respectively the receiver clock offset and its drift to be estimated. $a=e^{-T e / \tau}, T_{e}$ being the sampling time and $\tau$ the time constant of the auto-regressive model of bias $b^{j}(a \neq 0)$. The indexes of the vehicles and common satellites are respectively denoted by $i \in\left\{1 \ldots n_{r}\right\}$ and $j \in\left\{1 \ldots n_{s}\right\}$. For a practical implementation, the evolution model can be written in a discrete vectorial form ${ }^{i} \boldsymbol{x}_{k}=\boldsymbol{f}_{k}\left({ }^{i} \boldsymbol{x}_{k-1},{ }^{i} \boldsymbol{u}_{k-1}\right)$.

\section{B. Observability analysis}

Errors on pseudorange measurements are spatially correlated and common for close users [9]. Therefore, it is essential to study the observability to evaluate if the problem is solvable with no base station. Rife and Xiao [11] have shown that it is not possible to estimate biases simply by sharing GNSS pseudorange measurements between vehicles communicating in a Vehicle-to-Vehicle (V2V) network in a snapshot way (Epoch by Epoch). They have highlighted the limitation of distributing only GNSS data and proposed to add georeferenced information by using camera-based lane boundary sensor. A natural question that arises is whether GNSS biases are observable when adding vehicles motion information and errors evolution models.
In this section, we investigate the observability of the cooperative localization problem of vehicles sharing biases estimates when they are moving. The cooperative system described in section II given DR and pseudorange measurements is nonlinear. Therefore, we use the observability rank criterion based on Lie-Derivatives [12] to determine the conditions under which the system is locally weakly observable. Recently, Zhou et al. [13] have used the Lie derivatives to determine the conditions for the observability of $2 \mathrm{D}$ relative pose of pairs of mobile robots using range measurements. In the sequel, a test of Lie derivatives is considered for vehicles sharing GNSS errors. This study is inspired by the work of Zhou et al. [13]. Let consider $n_{r}$ vehicles and $n_{s}$ common visible satellites. Let $\hat{\boldsymbol{x}}$ be the estimated state of the cooperative system (S) as follows

$$
\hat{\boldsymbol{x}}=\left[\hat{\boldsymbol{p}}_{1}, \hat{d}_{1}, \ldots, \hat{\boldsymbol{p}}_{n_{r}}, \hat{d}_{n_{r}}, \hat{b}^{1}, \ldots, \hat{b}^{n_{s}}\right]^{T}
$$

with $\operatorname{dim}(\hat{\boldsymbol{x}})=4 n_{r}+n_{s} \cdot \hat{\boldsymbol{p}}_{1 \cdots n_{r}}$ are the 3D vehicles positions, $\hat{d}_{1 \cdots n_{r}}$ represent the receivers clock offsets. $\left(\hat{b}^{1 \cdots n_{s}}\right)$ denote the biases on $n_{s}$ common pseudorange measurements between vehicles.

The considered evolution model in this study consists in the first four DR equations of the system (3) and the last equation for the evolution of biases.

Let consider $u=\left[v_{1} \ldots v_{n_{r}}\right]^{T}$ the input of the system (S) in (4). The nonlinear DR model of (S) can be written as follows:

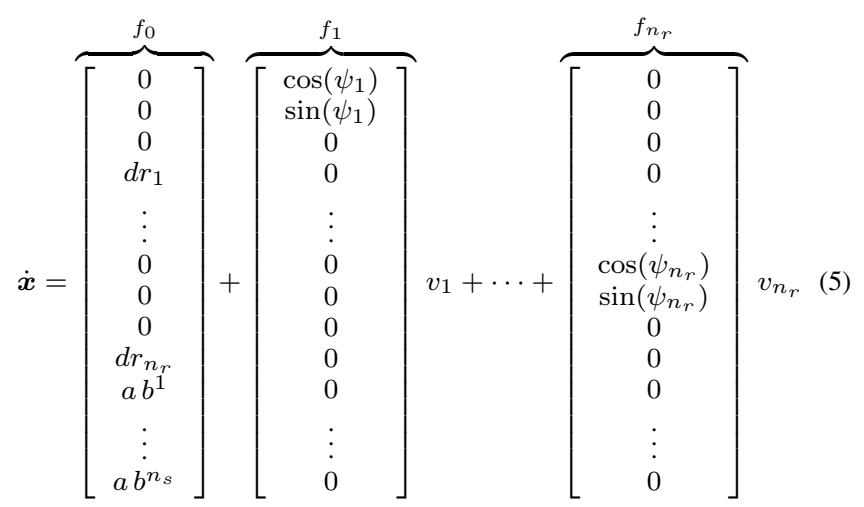

The nonlinear observation equations are given by

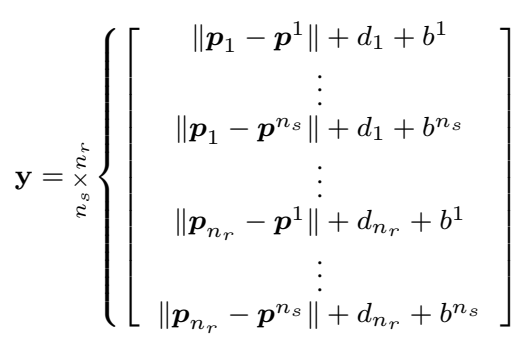

We compute hereafter the necessary Lie derivatives of $\mathbf{y}$ and their gradients:

Zeroth-order Lie derivatives $\left(\mathcal{L}^{0} \mathbf{y}\right)$ :

$$
\mathcal{L}^{0} \mathbf{y}=\mathbf{y}
$$

with gradient: 


$$
\begin{aligned}
& \nabla \mathcal{L}^{0} \mathbf{y}=j \operatorname{acobian}(\mathbf{y})
\end{aligned}
$$

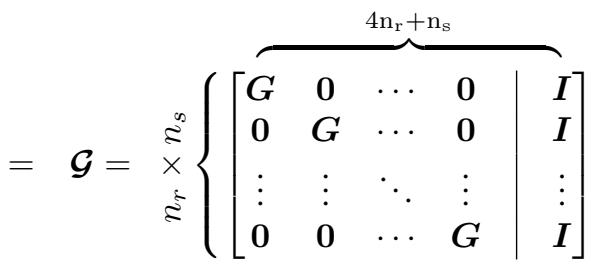

where $\boldsymbol{I}$ is the identity matrix with $\operatorname{dim}(\boldsymbol{I})=n_{s} \times n_{s}$ and $\mathcal{G}$ is the geometry matrix described in [11] where $G$ is defined as follows:

$$
\boldsymbol{G}=\left[\begin{array}{cc}
\left(\boldsymbol{u}^{1}\right)^{T} & 1 \\
\left(\boldsymbol{u}^{2}\right)^{T} & 1 \\
\vdots & \vdots \\
\left(\boldsymbol{u}^{n_{s}}\right)^{T} & 1
\end{array}\right]
$$

with $\operatorname{dim}\left(\boldsymbol{G}_{i}\right)=n_{s} \times 4$, the unit vector $\boldsymbol{u}^{j}$ in $\boldsymbol{G}$ is the estimated line of sight from the satellite $j$ to each user receiver $i$. This pointing vector is the same for all users when they are assumed to be in close proximity (e.g. distance between vehicles $\leq 10 \mathrm{~km}$ ):

$$
\boldsymbol{u}^{j}=\left(\boldsymbol{p}_{i}-\boldsymbol{p}^{j}\right) /\left\|\boldsymbol{p}_{i}-\boldsymbol{p}^{j}\right\| .
$$

First-order Lie derivatives $\left(\mathcal{L}_{f_{0}}^{1} \mathbf{y}\right)$ :

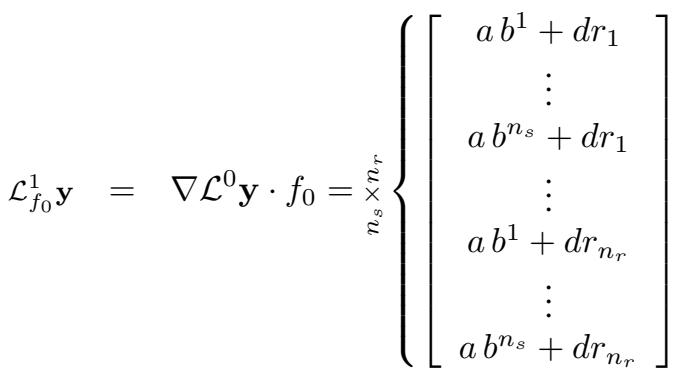

with gradient:

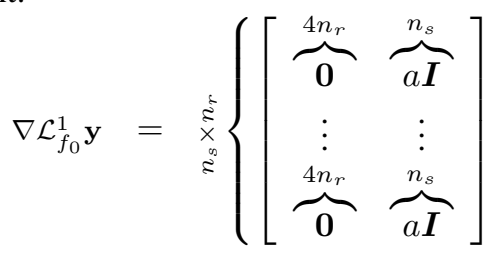

The observability matrix is now:

$$
\mathcal{O}=\left[\begin{array}{c}
\nabla \mathcal{L}^{0} \mathbf{y} \\
\nabla \mathcal{L}_{f_{0}}^{1} \mathbf{y}
\end{array}\right] .
$$

The role of the observability matrix in the observability analysis of a nonlinear system is given in [12], and recalled below.

Definition 1: Observability Rank Condition: The observability rank condition is satisfied when the observability matrix is full rank.

Theorem 1: Observability Necessary Condition: If the observability rank condition is satisfied for all values of the state $\boldsymbol{x}$, then the system is locally weakly observable.
Below, we compute the rank of the observability matrix (10) and determine the necessary conditions under which the system (S) is locally weakly observable.

$$
\operatorname{rank}(\mathcal{O})=\operatorname{rank}\left(\nabla \mathcal{L}^{0} \mathbf{y}\right)+\operatorname{rank}\left(\nabla \mathcal{L}_{f_{0}}^{1} \mathbf{y}\right)
$$

The rank of $\nabla \mathcal{L}^{0} \mathbf{y}$ has been studied in [11]. It has been proven that $\operatorname{rank}\left(\nabla \mathcal{L}^{0} \mathbf{y}\right)=4\left(n_{r}-1\right)+n_{s}$. It is straightforward to determine the rank of $\nabla \mathcal{L}_{f_{0}}^{1} \mathbf{y}$. Since the number of linearly independent equations in $\nabla \mathcal{L}_{f_{0}}^{1} \mathbf{y}$ appears to be $n_{s}$, $\operatorname{rank}\left(\nabla \mathcal{L}_{f_{0}}^{1} \mathbf{y}\right)=n_{s}$ if we have $a \neq 0$ (i.e. the biases change over time).

So, according to (11) we get:

$$
\operatorname{rank}(\mathcal{O})=4\left(n_{r}-1\right)+2 n_{s}
$$

The rank of $\mathcal{O}$ depends on the number $n_{s}$ of satellites in common among the users. It is obvious that $\operatorname{rank}(\mathcal{O})$ can not exceed the $n_{I}$ unknown states of (S) which is equal to $4 n_{r}+n_{s}$ (i.e. $\operatorname{rank}(\mathcal{O}) \leq 4 n_{r}+n_{s}$ ). So to get a full rank matrix, one must determine $n_{s}$ such that

$$
\operatorname{rank}(\mathcal{O}) \geq 4 n_{r}+n_{s} .
$$

By replacing (12) in (13) we get: $n_{s} \geq 4$. According to theorem 1, it can be concluded that the system is locally weakly observable regardless the number of users $\left(n_{r}\right)$ if the biases follow an auto-regressive model and if the vehicles have at least 4 common satellites.

\section{SET INVERSION WITH CSP}

Set inversion is a particular method of bounded error state estimation. This method avoids the data incest problem when fusing redundant data and gives reliable confidence domains of vehicles positions which is crucial for close navigation. Hereafter, some backgrounds of set inversion and CSP methods are recalled.

The objective of a set inversion method is to determine the unknown state $\mathbb{X} \subset \mathbb{R}^{n}$ such that $\mathbf{f}(\mathbb{X}) \subset \mathbb{Y}$, where $\mathbb{Y}$ is a given set of measurements. We have then to compute the reciprocal image $\mathbb{X}=\mathbf{f}^{-1}(\mathbb{Y})$. A guaranteed approximation of the solution set $\mathbb{X}$ can be done using 2 sub-pavings which bracket the solution set as follows $\mathbb{X} \subset \mathbb{X} \subset \overline{\mathbb{X}}$ (Fig. 2). A box $[\boldsymbol{x}]$ of $\mathbb{R}^{n}$ is feasible if it is inside $\mathbb{X}$ and unfeasible if it is outside $\mathbb{X}$, otherwise $[\mathbf{x}]$ is indeterminate. SIVIA, used in this work, is an algorithm for solving a set inversion problem using interval analysis by testing feasibility of boxes [3], [14]. It explores an arbitrarily large initial box by testing its inclusion or not in $\mathbb{Y}$ using an inclusion function $[\mathbf{f}]$ and bisecting it otherwise. However, a bisection increases exponentially the complexity of the computation, particularly when treating box of a high dimension problem. In order to solve this problem, CSP is used to reduce the box in an optimal way before being bisected. A CSP aims at solving a set of constraints denoted by $\mathcal{H}$ (Eq.14), in which a box $[\boldsymbol{x}]$ should satisfy all the constraints of a vector $F$.

$$
C S P \mathcal{H}:\{(F(x)=\mathbf{0} \mid x \in[x])\}
$$


Contracting $\mathcal{H}$ means replacing $[\mathbf{x}]$ by a smaller domain $\left[\mathbf{x}^{\prime}\right]$ such that the solution set remains unchanged. The used contractor strategy consists in Forward-Backward Propagation [15]. The CSP consists then in applying contractors using Waltz's algorithm [16]. CSP and SIVIA algorithm can be mixed together to get the "SIVIAP" algorithm (please refer to [6], [14]) in order to speed up the processing by reducing the number of bisections through boxes contractions.

\section{Cooperative Localization with Reliable CONFIDENCE DOMAINS}

The cooperative localization strategy is similar to the one presented in [10], where vehicles share their estimated GNSS errors, DR measurements and positions in order to improve the absolute and relative positioning. The main difference consists in using the set-membership method, in particular the set inversion method under CSP, instead of the Bayesian one. The objective is to get reliable confidence domains that contain the true positions of the vehicles.

\section{A. Problem statement}

Let us describe the variables, the domains and the constraints of the considered CSP.

Variables. In each agent $R_{i}$, there is an ego state to be estimated and tracked poses of the other vehicles $R_{o}$ in the group, where $o \in\left\{1, \ldots, n_{r}-1\right\}$ and $o \neq 0$.

Ego state: let $\nu=\left\{x_{i}, y_{i}, z_{i}, b^{1}, \ldots, b^{n_{s}}, d_{i}, \dot{d}_{i}\right\}$ be the variables of the ego state ${ }^{i} \boldsymbol{x}_{\text {ego }}$ of $R_{i}$ of dimension $n=$ $5+n_{s}$. Only these variables are considered in the CSP. We denote the absolute pose by ${ }^{i} \boldsymbol{q}=\left\{x_{i}, y_{i}, z_{i}\right\}$, the biases of all $n_{s}$ satellites in view by ${ }^{i} \boldsymbol{b}=\left\{b^{1}, \ldots, b^{n_{s}}\right\}$ and the inner variables of $R_{i}$ by ${ }^{i} \boldsymbol{\xi}=\left\{d_{i}, \dot{d}_{i}\right\}$. which contain respectively the clock offset $d_{i}$ and its drift $\dot{d}_{i}$.

Tracked pose: let ${ }^{i} \boldsymbol{q}_{o}=\left\{{ }^{i} \boldsymbol{q}_{1}, \ldots,{ }^{i} \boldsymbol{q}_{n_{r}-1}\right\}$ be the pose of the other vehicles.

The ego input of $R_{i}$ is denoted by ${ }^{i} \boldsymbol{u}_{e g o}=\left\{v_{i}, \psi_{i}\right\}$, where $v_{i}$ and $\psi_{i}$ are respectively the linear speed and the heading angle measurements. ${ }^{i} \boldsymbol{u}_{o}=\left\{{ }^{i} \boldsymbol{u}_{1}, \ldots,{ }^{i} \boldsymbol{u}_{n_{r}-1}\right\}$ represents the input of $R_{o}$ composed of ${ }^{i} \boldsymbol{u}_{o}=\left\{v_{o}, \psi_{o}\right\}$.

Domains. The domains of the variables are sets enclosing the true value of the variables which are presented as intervals. For example, the states of the vehicles are vectors of intervals of $\mathbb{R}^{n}$

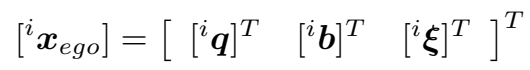

Constraints. Let us now describe the constraints which link the variables at each time $k$ as (i) ${ }^{i} x_{e g o, k}=$ $\boldsymbol{f}\left({ }^{i} \boldsymbol{x}_{\text {ego }, k-1},{ }^{i} \boldsymbol{u}_{\text {ego }}\right)$ and (ii) ${ }^{i} \boldsymbol{y}_{k}=\boldsymbol{g}\left({ }^{i} \boldsymbol{x}_{\text {ego }, k}\right)$. The constraint (i) corresponds to the evolution equation. The constraint (ii) defines the CSP which is used in SIVIAP. $\boldsymbol{g}$ is replaced by its natural inclusion function, ${ }^{i} \boldsymbol{y}_{k}$ corresponds to measurements intervals to be inverted and ${ }^{i} \boldsymbol{x}_{e g o, k}$ is a prior feasible box.

Solver. SIVIAP approximates the vector state ${ }^{i} \boldsymbol{x}_{e g o, k}$ such that $\boldsymbol{g}\left({ }^{i} \boldsymbol{x}_{\text {ego }, k}\right) \subseteq\left[{ }^{i} \boldsymbol{y}_{k}\right]$ using the forward-backward contrac-

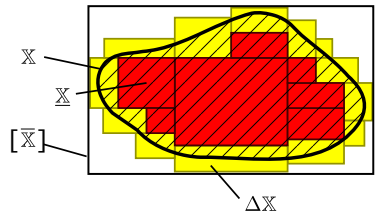

Fig. 2: Bracketing of solution set $\mathbb{X}$ between two subpavings $\mathbb{X}$ and $\overline{\mathbb{X}}=\underline{\mathbb{X}} \cup \Delta \mathbb{X} \cdot[\overline{\mathbb{X}}]$ is the covering box of $\overline{\mathbb{X}}$.

tors. The set CSP to be characterized by SIVIAP is defined below (Eq. 15)

$$
\begin{aligned}
& C S P=\left\{{ }^{i} \boldsymbol{x}_{\text {ego }, k} \subseteq\left[{ }^{i} \boldsymbol{x}_{\text {ego }, k}\right] \backslash \boldsymbol{g}\left({ }^{i} \boldsymbol{x}_{\text {ego }, k}\right) \subseteq\left[{ }^{i} \boldsymbol{y}_{k}\right]\right\} \\
& =\boldsymbol{g}^{-1}\left(\left[{ }^{i} \boldsymbol{y}_{k}\right]\right) \cap\left[{ }^{i} \boldsymbol{x}_{e g o, k}\right]
\end{aligned}
$$

We describe the distributed cooperative Algorithm 1 in any vehicle $R_{i}$.

Agent $R_{i}$ predicts its ego state ${ }^{i} \boldsymbol{x}_{\text {ego }}$ using the evolution model in Eq. (3) and its DR inputs $\left(v_{i}, \psi_{i}\right)$ measured at every $0.01 \mathrm{~s}$ (line 1 ). Moreover, each vehicle $i$ tracks the other vehicles (line 2) using their last received DR inputs and the discrete evolution function of the position which is presented in its continuous form in Eq. (3).

Lines $(4 \cdots 14)$ of the algorithm consist in updating the predicted state ${ }^{i} \boldsymbol{x}_{\text {ego }}$ with respect to the GNSS measurements which are available every $0.2 s$. In order to reject the outliers at each time $k$ when the GNSS measurements are available, a validation process on the measurements of every satellite is performed. For every pseudorange measurement, we check if the $S N R$ (Signal to Noise Ratio) of the satellite is high enough (e.g. $35 \mathrm{~dB} / \mathrm{Hz}$ ). Afterward, we apply the SIVIAP algorithm presented in [14] with the following modifications. The considered solution is the covering box of $\overline{\mathbb{X}}$, which is the union of the inner $\mathbb{X}$ and indeterminate $\Delta \mathbb{X}$ subpavings as it is shown in Fig 2. In order to stop the bisections, we limit the computational time at $0.1 s$ for each GNSS pseudorange measurement epoch.

In our problem, the delays of communication (line 15) have to be smaller than the processing period. If not, steps 16 and 17 of the algorithm can be extended with data buffers to do the prediction on a horizon. The sent (dataS) and received (dataR) information at each instant $k$ by each vehicle are its last ego pose, its last estimates of the biases, its current DR input vector and its identifier $i d$ in the group. The amount of the transmitted information is low, since vehicles only exchange the lower and upper bounds of bounding boxes of the subpavings.

In line 16 of the algorithm, each vehicle $i$ updates the tracked pose of the others $R_{o}$ by the received estimated pose $\left[{ }^{i} \boldsymbol{q}_{o}\right]:=\left[{ }^{o} \boldsymbol{q}_{\text {ego }}\right]$. Finally, $R_{i}$ merges its estimated biases with the received ones from the other vehicles $R_{o}$ simply as the intersection $\left[{ }^{i} \boldsymbol{b}\right]=\left[{ }^{i} \boldsymbol{b}\right] \cap\left[{ }^{o} \boldsymbol{b}\right]$.

\section{EXPERIMENTAL RESULTS}

We present the results of two scenarios - Standalone (S) and Cooperative $(\mathrm{C})$ - to quantify the performance gain due to the cooperation. The proposed approach has been tested with two experimental vehicles (Fig. 1). The data-set is the 

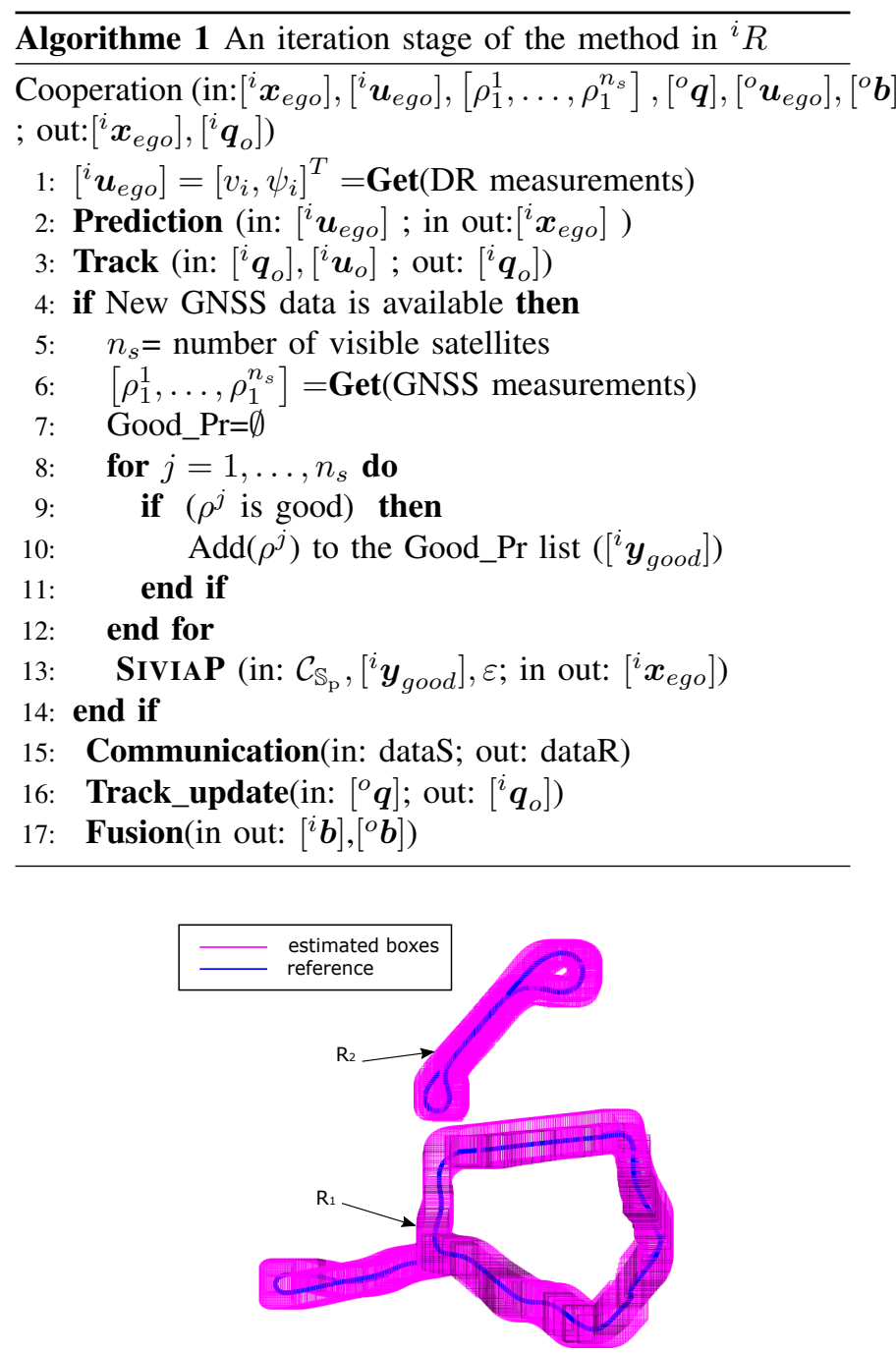

Fig. 3: Trajectories and position boxes of both vehicles.

same as the one used in [10]. A low-cost U-blox $4 T$ GPS receiver providing pseudoranges was used in every vehicle. A Polarx Septentrio was used in RTK mode to obtain the ground truth data and a CAN-bus gateway to get the DR measured input $\boldsymbol{u}_{\text {ego }}=\left[\begin{array}{ll}v & \psi\end{array}\right]^{T}$. 10 satellites were in view during the test and 5 of them were at least in common which satisfies the necessary condition of the observability discussed in section II-B. The GPS satellite visibility was sometimes very constrained due to the buildings and trees near the test area since the experiments were carried out in the campus of the university. The reported test was around 4 minutes long. The algorithm has been implemented in $\mathrm{C}++$ using the interval library IBEX [17]. Fig. 3 shows a top view of the trajectories and the estimated position boxes of both vehicles using the (C) method. The representation of solution is the covering box $[\overline{\mathbb{X}}]$ of the union of the inner and indeterminate subpavings: $\mathbb{X} \cup \Delta \mathbb{X}$.

Fig. 4 and 5 show the bounds of position errors of the vehicles respectively for the $x$ and $y$ dimensions using the (C) and $(\mathrm{S})$ methods. At a first glance, one can check the integrity of both observers due to the fact that bounds contain always the zero value. It means that the RTK reference position
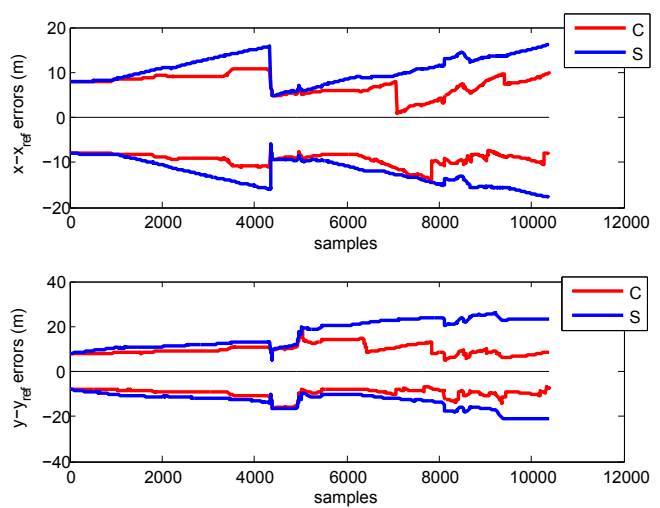

Fig. 4: Bounds of position errors of $R_{1}$.
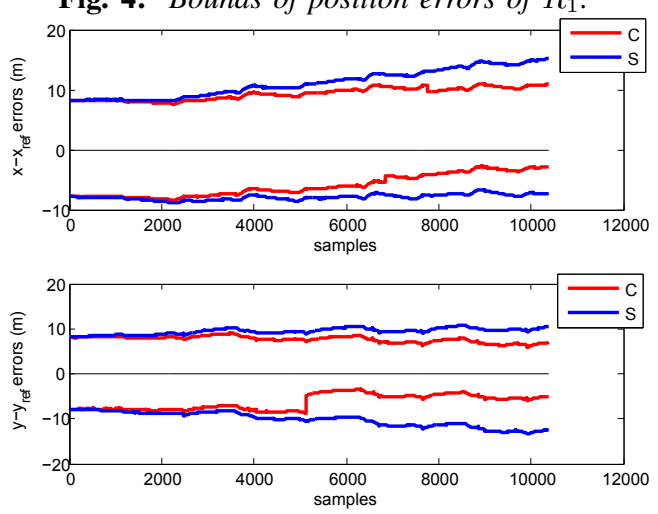

Fig. 5: Bounds of position errors of $R_{2}$.

is always included in the estimated boxes (i.e. guaranteed results) which indicates a good tuning of the observers.

Fig. 6 shows the estimated bounds of the relative distance. Thanks to the cooperation, there is gain of accuracy in the order of several meters.

Table I gives some performance metrics for both methods in term of absolute and relative HPE and Confidence Domain Size (CDS) of the resulting boxes. The CDS is simply the surface of the 2D boxes here. The Cumulative Distribution Functions (CDFs) of the CDS of the vehicles boxes throughout the trajectory are shown in Fig. 7. A net improvement is obtained for vehicle $R_{1}$ in terms of accuracy and uncertainty reduction. For instance, the median of the HPE is reduced from $2.41 \mathrm{~m}$ to $1.67 \mathrm{~m}$ and the $\mathrm{CDS}$ is $66 \%$ condensed due to the cooperation since the 95th percentile of CDS is less than $466.6 \mathrm{~m}^{2}$ compared to $1390 \mathrm{~m}^{2}$ when using the $\mathrm{S}$ method. Concerning vehicle $R_{2}$, the improvement of the HPE is not as noticeable as for $R_{1}$. Indeed, $R_{2}$ used more satellites for the biases update than $R_{1}$. This explains the improvement of the positioning performance of $R_{1}$ compared to $R_{2}$. Nevertheless, the uncertainty is reduced a lot as since the 95th percentile of the CDS is less than $256 \mathrm{~m}^{2}$ compared to $501.4 \mathrm{~m}^{2}$ when using the $\mathrm{S}$ method which is a $49 \%$ improvement.

Regarding the relative HPE, the method has improved the accuracy of the relative localization thanks to the fusion of the biases estimates. Especially, if we look at the error average and error standard deviation, there are reduced respectively from $3.66 \mathrm{~m}$ and $2.87 \mathrm{~m}$ to $3.01 \mathrm{~m}$ and $2.46 \mathrm{~m}$. 


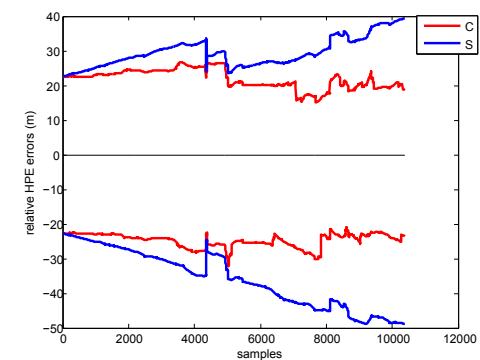

Fig. 6: Bounds of the relative distance between the vehicles

\begin{tabular}{|c|c|c|c|c|}
\hline \multicolumn{2}{|c|}{} & HPE R1 & HPE R2 & Relative HPE \\
\hline \hline \multirow{2}{*}{ Std. dev. $\left(m^{2}\right)$} & $\mathrm{C}$ & 1.69 & 1.41 & 2.46 \\
\cline { 2 - 5 } & $\mathrm{S}$ & 2.28 & 1.33 & 2.87 \\
\hline \multirow{2}{*}{ Max $(m)$} & $\mathrm{C}$ & 6.02 & 4.30 & 8.87 \\
\cline { 2 - 5 } & $\mathrm{S}$ & 5.77 & 4.28 & 8.27 \\
\hline \multirow{2}{*}{ Median $(m)$} & $\mathrm{C}$ & 1.67 & 1.95 & 3.01 \\
\cline { 2 - 5 } & $\mathrm{S}$ & 2.41 & 1.76 & 3.66 \\
\hline
\end{tabular}

TABLE I: $H P E$ and $C D S$ statistics

The bias on every pseudorange has been initialized with the interval $[-30,30]$ giving no prior knowledge. For each subplot of Fig. 8, the $x$ axis expresses the number of samples, the $y$ axis gives every center and bounds of the estimated bias interval $\left[b^{j}\right]$ in meters where $j=1, \ldots, n_{s}$. Note that all subplots are truncated in order to observe the convergence illustrated by the horizontal final asymptotes. This convergence highlights the usefulness of the previous observability analysis. The observed final values of the biases are very common for a low cost GNSS receiver.

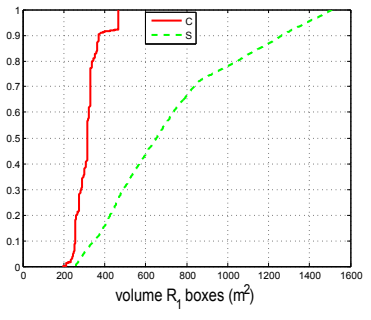

(a) $C D S$ of $R_{1}$

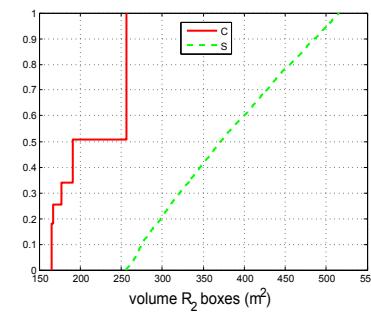

(b) $C D S$ of $R_{2}$
Fig. 7: Cumulative Distribution of Confidence Domain Size (CDS) VI. CONCLUSION

A cooperative localization method for intelligent vehicles sharing GNSS common errors has been proposed, studied and evaluated experimentally. Thanks to an observability study, it has been found that at least 4 satellites and an auto-regressive model of biases are needed to keep the states observable. Afterward, a new distributed cooperative approach using a set inversion method with CSP has been proposed. This method has the advantage to guarantee that there is no data incest and it handles rigorously the nonlinearity of the equations. This new cooperative method provides a significant enhancement to absolute and relative positioning in terms of accuracy and confidence domains compared to usual standalone methods.

\section{ACKNOWLEDGMENT}

This work has been carried out within the framework of the Equipex ROBOTEX (Reference ANR-10- EQPX-44-01).
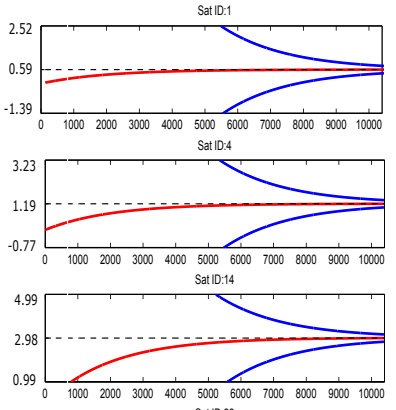
Sat ID:22
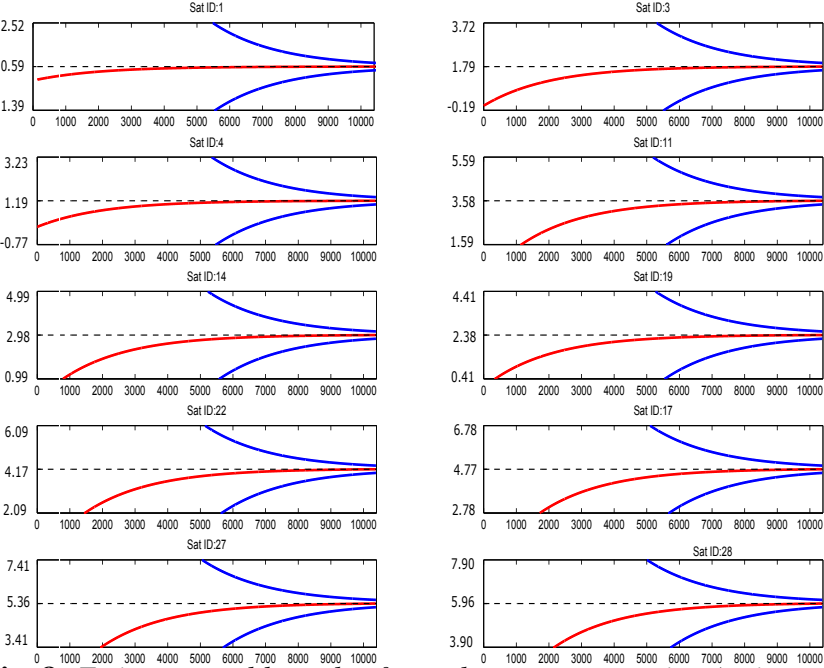

Satlo:

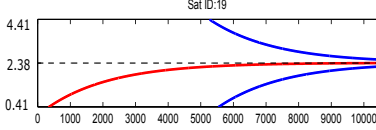
5000 at ID:17
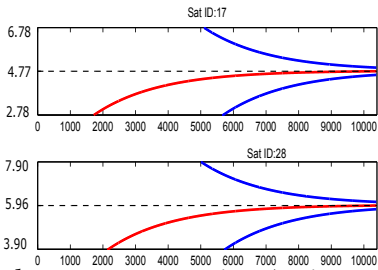

Fig. 8: Estimates and bounds of pseudoranges correction (units are samples and meters).

\section{REFERENCES}

[1] N. Karam, F. Chausse, R. Aufrere, and R. Chapuis. Localization of a group of communicating vehicles by state exchange. In IEEE Int Conf on Intelligent Robots and Systems, pages 519-524, Oct 2006.

[2] A Ahmad, G.D. Tipaldi, P. Lima, and W. Burgard. Cooperative robot localization and target tracking based on least squares minimization. In Int Conf on Robotics and Automation, pages 5696-5701, 2013.

[3] Jaulin L. and Walter E. Set inversion via interval analysis for nonlinear bounded-error estimation. Automatica, 29(4):1053-1064, 1993.

[4] V. Drevelle and Ph. Bonnifait. Localization Confidence Domains via Set Inversion on Short-Term Trajectory. IEEE Trans on Robotics, 29(5):1244-1256, 2013.

[5] D. Meizel, O. Leveque, L. Jaulin, and E. Walter. Initial localization by set inversion. IEEE Trans on Robotics and Automation, 18(6):966971, 2002.

[6] L. Jaulin. Interval constraint propagation with application to boundederror estimation. Automatica, 36(10):1547-1552, 2000.

[7] V. Drevelle, L. Jaulin, and B. Zerr. Guaranteed characterization of the explored space of a mobile robot by using subpavings. In IFAC Symposium on Nonlinear Control Systems, pages 44-49, Sept 2013.

[8] A. Bethencourt. Interval Analysis for swarm localization. Application to underwater robotics. PhD thesis, University of Bretagne, Sept 2014.

[9] E. D. Kaplan and C. Hegarty. Understanding GPS: Principles and Applications. Second Edition, Artech House, 2005.

[10] K. Lassoued, I. Fantoni, and P. Bonnifait. Mutual localization and positioning of vehicles sharing GNSS pseudoranges: Sequential bayesian approach and experiments. In IEEE Int Conf on Intelligent Transportation Systems, Spain, Sept 2015.

[11] X. Xiao J. Rife. Estimation of spatially correlated errors in vehicular collaborative navigation with shared GNSS and road-boundary measurements. In Int Technical Meeting of The Satellite Division of the Institute of Navigation, pages 1667-1677, Sept 2010.

[12] R. Hermann and A.J. Krener. Nonlinear controllability and observability. IEEE Trans on Automatic Control, 22(5):728-740, Oct 1977.

[13] X.S. Zhou and S.I. Roumeliotis. Robot-to-robot relative pose estimation from range measurements. IEEE Trans on Robotics, 24(6):13791393, Dec 2008.

[14] L. Jaulin, M. Kieffer, O. Didrit, and E. Walter. Applied interval analysis with examples in parameter and state estimation, robust control and robotics. Springer, 2001.

[15] K. Lassoued, O. Stanoi, Ph. Bonnifait, and I. Fantoni. Mobile robots cooperation with biased exteroceptive measurements. In Int Conf on Control Automation Robotics Vision, pages 1835-1840, 2014.

[16] D. Waltz. Generating semantic descriptions from drawings of scenes with shadows. The psychology of computer vision, 1975.

[17] G. Chabert. IBEX(http://www.ibex-lib.org), 2007. 